\title{
First passage properties of a generalized Pólya urn
}

\author{
Michael J Kearney ${ }^{1}$ and Richard J Martin ${ }^{2}$ \\ ${ }^{1}$ Senate House, University of Surrey, Guildford, Surrey, GU2 7XH, UK \\ ${ }^{2}$ Department of Mathematics, Imperial College London, South Kensington, \\ London, SW7 2AZ, UK
}

\begin{abstract}
A generalized two-component Pólya urn process, parameterized by a variable $\alpha$, is studied in terms of the likelihood that due to fluctuations the initially smaller population in a scenario of competing population growth eventually becomes the larger, or is the larger after a certain passage of time. By casting the problem as an inhomogeneous directed random walk we quantify this role-reversal phenomenon through the first passage probability that equality in size is first reached at a given time, and the related exit probability that equality in size is reached no later than a given time. Using an embedding technique, exact results are obtained which complement existing results and provide new insights into behavioural changes (akin to phase transitions) which occur at defined values of $\alpha$.
\end{abstract}

\footnotetext{
Emails: $\quad$ m.j.kearney@ surrey.ac.uk richard.martin1@imperial.ac.uk

Keywords: Stochastic processes, growth processes, critical phenomena of socioeconomic systems
} 


\section{Contents}

1. Introduction

2. Dynamical embedding

3. Calculations

3.1 The exit probability

3.2 The survival probability

3.3 The role-reversal probability

3.4 The first passage probability

3.5 Special cases

4. Discussion

Acknowledgements

Appendix A. Birth processes

Appendix B. Asymptotic normality

Appendix C. The standard Pólya urn

References

\section{Introduction}

A generalized two component Pólya urn, whose individual integer populations $A, B$ evolve stochastically in discrete time, may be defined by, 


$$
\begin{aligned}
& \operatorname{Pr}((A, B) \rightarrow(A+1, B))=\frac{A^{\alpha}}{A^{\alpha}+B^{\alpha}} \\
& \operatorname{Pr}((A, B) \rightarrow(A, B+1))=\frac{B^{\alpha}}{B^{\alpha}+A^{\alpha}}
\end{aligned}
$$

for a given exponent $\alpha$ and initial condition $(A, B)=(a, b)$. For the choice $\alpha=1$ this is simply the standard Pólya urn process [1,2], which arises naturally in the physical, life and social sciences in areas such as evolutionary theory, epidemiology and computer informatics [3, 4]. For the choice $\alpha=-1$ one has instead the Friedman urn process $[5,6]$ which appears in the analysis of competition and conflict $[7,8]$. Given this ubiquity, the generalization through (1) suggests itself and similar constructs arise in the context of clinical trials [9], neuronal development [10], the organization of growing networks exhibiting preferential attachment [11-13] and the emergence of macrostructure in economics $[14,15]$. If we think of (1) as a model of competing population growth, then inherent in the model for $\alpha>0$ is the idea of growth reinforcement, a tendency colloquially referred to as 'the rich get richer'.

The main topic of focus in this paper is to study the opposite tendency, namely the likelihood that, given an initial population imbalance, the smaller population eventually becomes the larger, or is the larger after a certain time. The issue of 'rolereversal' of population sizes (and related to it the issue of the time to first achieve equality) is of interest in many of the aforementioned areas of application [16]. This fluctuation-driven phenomenon has been studied for the standard Pólya urn process using conventional combinatorial techniques [17]. The corresponding analysis when $\alpha \neq 1$ requires a different approach. 
One can represent the process evolving under (1) as a directed random walk or lattice path on a two-dimensional lattice whose axes correspond to the populations $A$ and $B$; see figure 1 . Starting from a point $(a, b)$, where without loss of generality we assume $a>b$ throughout the paper, the question of role-reversal is then equivalent to studying if or when a given path crosses the line $B=A$. We characterize this (using the terminology of [17]) by calculating the first passage probability $G_{n}(a, b)$ that a path of the process reaches the line $B=A$ for the first time precisely at the point $(n, n)$, and the related exit probability $E_{n}(a, b) \equiv \sum_{j=a}^{n} G_{j}(a, b)$, which is defined as the probability that a given path reaches the line $B=A$ no later than the point $(n, n)$. The survival probability $\bar{E}_{n}(a, b) \equiv 1-E_{n}(a, b)$ provides a measure of persistence with respect to the time-like parameter $n$; in addition, following appropriate manipulation the exit probability yields the role-reversal probability $P_{S}(B>A)$ that after a given number of steps $S$ the population that was initially the smaller is now the larger. For a general overview of first passage processes and persistence see [18, 19].

It is known that the standard Pólya urn process $(\alpha=1)$ is transient, which means that a given path as described above is not guaranteed to cross the line $B=A$ (in other words, if we consider the population difference variable $\Delta \equiv A-B$ evolving as a function of the number of steps $S$ then there is a non-zero probability that $\Delta$ will not reach zero as $S \rightarrow \infty)$. On the other hand, the isotropic random walk $(\alpha=0)$ is recurrent, which means that a given path is guaranteed to eventually cross the line $B=A$ (the term 'isotropic' is appropriate in the sense that, with each step, $\Delta$ increases or decreases with equal probability $\frac{1}{2}$ when $\alpha=0$ ). Thus the behaviour changes qualitatively at some critical value $0<\alpha<1$ and the critical case turns out to 
be $\alpha=\frac{1}{2}$ (which itself is marginally recurrent). The analysis of the quantities $G_{n}(a, b)$ and $E_{n}(a, b)$ illustrates this transition in behaviour at $\alpha=\frac{1}{2}$ in a new and interesting way.

The long-time asymptotic dynamics under (1) has been studied previously using special embedding techniques (which we later adapt to our present purpose) and it is already known that as the number of steps or the elapsed time $S \rightarrow \infty$ the following occurs almost surely [10, 20-22]:

1. If $\alpha \leq \frac{1}{2}$, both populations tend to infinity but, in doing so, alternate as to which is the largest arbitrarily many times;

2. If $\frac{1}{2}<\alpha \leq 1$, both populations tend to infinity but beyond a certain (realization specific) time one is always larger than the other;

3. If $\alpha>1$, one of the populations tends to infinity whilst the other remains finite.

The three distinct regimes, in order, are referred to as almost balanced, eventual leadership and monopoly. Such marked changes in behaviour (akin to phase transitions) occurring at $\alpha=1$ as well as at $\alpha=\frac{1}{2}$ demonstrate the richness of the model. The standard Pólya urn $(\alpha=1)$ exhibits marked sensitivity to initial conditions and large realization-to-realization fluctuations, a key property being that the ratio $A / B$ approaches a well-defined limiting value but one which is realization specific $[3,4]$. The results here extend to general $\alpha$ what is known as regards the dependency on initial conditions as well as the system state at a given finite time. 
The representation of the process in terms of the time evolution of the population difference variable $\Delta$ highlights a close connection between the dynamics under (1) and a model of history-dependent (long-range correlated) random walks which finds application in characterizing financial data, coarse grained DNA strings and even written texts $[23,24]$. Characteristically these walks exhibit super-diffusive behaviour when a parameter exceeds a certain value (corresponding to $\alpha=\frac{1}{2}$ in the present case). The link with the Pólya and Friedman urn processes was pointed out in [25]; the present work provides fresh insights.

The paper is structured as follows. In Section 2, an embedding procedure is introduced based on continuous-time birth processes. This is used in Section 3 to calculate exact results for any $\alpha$ for various quantities, in particular the exit probability $E_{n}(a, b)$ and the first passage probability $G_{n}(a, b)$. The underlying mathematical structure of the transition at $\alpha=\frac{1}{2}$ is made transparent using an approximation scheme based on the asymptotic normality of the underlying birth processes. Comparison is also made with the results of simulations, with excellent agreement. By setting $\alpha=1$ certain known results for the standard Pólya urn process are re-derived, but in a quite different form. Finally, in Section 4, the results are discussed and avenues for further work are identified.

\section{Dynamical embedding}

As discussed above, a given realisation or lattice path of the process starts from the point $(a, b)$ and, after a fixed number of steps $S$ (the elapsed time), ends at the point $(A, B)$ which lies on the line $A+B=a+b+S$ (see figure 1 ). The calculation of the 
various quantities of interest involves summing over a defined set of paths, but conventional combinatorial arguments cannot be used (except for the special cases $\alpha=0$ and $\alpha=1$ ) since for general $\alpha$ paths between two given points do not necessarily have the same probabilistic weight (e.g. compare the weights of the two paths between the points $(a, b)$ and $(a+1, b+1)$ for $a>b)$. This is a significant complicating factor which requires a different approach to the problem.

To this end, we adapt the embedding techniques used in [10, 20-22] which draw on the theory of branching processes [26]. The basic idea was seemingly first described in [27], then developed further in [28, 29] and has been reviewed in [30, 31]. Let $X(t)$ be a birth process $[26,32]$ on the non-negative integers $j$ with statedependent transition rates $j^{\alpha}$, and suppose $X(0)=a$. Let $Y(t)$ be an independent version of the same process with $Y(0)=b$, and consider the probability that $X(t)$ makes the jump from $a$ to $a+1$ before $Y(t)$ makes the jump from $b$ to $b+1$. Using elementary properties of birth processes (see Appendix A) we have,

$$
\operatorname{Pr}((a, b) \rightarrow(a+1, b))=\int_{0}^{\infty} a^{\alpha} e^{-a^{\alpha} T} \times e^{-b^{\alpha} T} d T=\frac{a^{\alpha}}{a^{\alpha}+b^{\alpha}}
$$

and correspondingly $\operatorname{Pr}((a, b) \rightarrow(a, b+1))=b^{\alpha} /\left(b^{\alpha}+a^{\alpha}\right)$. With reference to (1) we see that this generates the correct transition probabilities for the first step of the process. Since birth processes are memoryless, it follows that if we track the time evolution of $X(t)$ and $Y(t)$ then the time-ordered sequence in which the jumps occur corresponds uniquely to a lattice path and has the same probabilistic weight; see figure 2. One can therefore sum over defined sets of lattice paths by integrating over 
all possible realisations of $X(t)$ and $Y(t)$ consistent with the given boundary

conditions. The elegant feature is that the independence of $X(t)$ and $Y(t)$ makes this a relatively straightforward task. All one requires are two quantities for $X(t)$ (and the corresponding quantities for $Y(t))$, namely the probability $P(a, k ; t)$ of being in state $k$ at time $t$ and the closely related probability density function $p(a, k ; T)$ for the first passage time $T$ to reach state $k$. These are derived in Appendix A.

\section{Calculations}

\subsection{The exit probability}

One can use these ideas to calculate the first passage probability $G_{n}(a, b)$ and the exit probability $E_{n}(a, b) \equiv \sum_{j=a}^{n} G_{j}(a, b)$, as defined in the Introduction. It turns out that it is actually easiest to calculate $E_{n}(a, b)$ directly without first having to calculate $G_{n}(a, b)$; the latter then follows naturally since $G_{n}(a, b)=E_{n}(a, b)-E_{n-1}(a, b)$. Let us consider paths on the lattice where the lines $A=n+1$ and $B=n+1$ are taken to be absorbing. This is a purely technical device and when required we will take the limit $n \rightarrow \infty$. Let us consider in particular all the paths which start at $(a, b)$ where $a>b$

and end on the absorbing line $B=n+1$ rather than the absorbing line $A=n+1$. Each such path must necessarily reach the line $B=A$ for the first time at some point $(k, k)$ where $a \leq k \leq n$; see figure 1 . By symmetry, for each such path there is also a reflected path from this point onwards, of equal probabilistic weight, which ends on the line $A=n+1$ rather than the line $B=n+1$. Therefore, by summing over all the paths which end on the line $B=n+1$ one captures precisely half the probabilistic 
weight of all the paths that reach the line $B=A$ no later than the point $(n, n)$. The latter, of course, is $E_{n}(a, b)$, the quantity we seek, and thus to calculate it one simply has to integrate over realisations for which at the time the process $Y(t)$ makes the transition to state $n+1$ the process $X(t)$ is not in state $n+1$, and multiply by a factor of two;

$$
E_{n}(a, b)=2 \int_{0}^{\infty} p(b, n+1 ; T)[1-P(a, n+1 ; T)] d T .
$$

Using (A4) and (A6) in Appendix A one obtains after carrying out the integral and simplifying using the partial fraction identity (A8),

$$
E_{n}(a, b)=2 \sum_{j=a}^{n}\left\{\prod_{\substack{\ell=a \\ \ell \neq j}}^{n} \frac{\ell^{\alpha}}{\ell^{\alpha}-j^{\alpha}}\right\}\left\{\prod_{\ell^{\prime}=b}^{n} \frac{\ell^{\prime \alpha}}{\ell^{\prime \alpha}+j^{\alpha}}\right\} ; \quad \alpha \neq 0 .
$$

This result is exact. One can check that $E_{n}(a, b)$ satisfies, based on an elementary decomposition, the recurrence equation;

$$
E_{n}(a, b)=\frac{a^{\alpha}}{a^{\alpha}+b^{\alpha}} E_{n}(a+1, b)+\frac{b^{\alpha}}{b^{\alpha}+a^{\alpha}} E_{n}(a, b+1)
$$

with $E_{n}(a, a)=1$ for $n \geq a$. This admits an obvious interpretation. The exact expression for $E_{n}(a, b)$ for the special case where $\alpha=0$ is given in Section 3.4. 
One can derive another integral representation for the exit probability. Consider all realisations for which, at the time the process $X(t)$ makes the transition to the absorbing state $n+1$, the process $Y(t)$ is already in the absorbing state $n+1$. All such realisations have the property that at the earlier time the process $Y(t)$ reached state $n+1, X(t)$ was not in state $n+1$; see figure 2 . This observation in conjunction with earlier arguments leads to,

$$
\begin{aligned}
E_{n}(a, b) & =2 \int_{0}^{\infty} p(a, n+1 ; T) P(b, n+1 ; T) d T \\
& =2 \int_{0}^{\infty} p(a, n+1 ; T) \int_{0}^{T} p\left(b, n+1, T^{\prime}\right) d T^{\prime} d T
\end{aligned}
$$

where the last step uses the relationship given below (A6) in Appendix A. This result plays an important role below. By setting $b=a$, integrating by parts and exploiting the normalisation of $p(a, n+1 ; T)$ one has a quick check that $E_{n}(a, a)=1$.

In figure 3 , a comparison is made between $E_{n}(a, b)$ based on numerical simulations using the local rules (1) and the exact result (4), in this instance plotted as a function of $n$ for various values of $\alpha$. The agreement is excellent. The results given in the Introduction imply that $E_{n}(a, b) \rightarrow 1$ as $n \rightarrow \infty$ for $\alpha \leq \frac{1}{2}$ whilst $E_{n}(a, b)$ saturates to a value $E_{\infty}(a, b)<1$ for $\alpha>\frac{1}{2}$. One cannot easily infer this from (4) but we can justify it using a scheme for making asymptotically accurate approximations based on the integral representation (6). 
The key idea is that the first passage time $T$ for the birth process $X(t)$ to reach state $n+1$ starting from state $a$ is asymptotically normal. Let us write quite generally,

$$
p(a, n+1 ; T)=\frac{1}{\sqrt{2 \pi \sigma^{2}(a, n+1)}} \exp \left\{-\frac{(T-\mu(a, n+1))^{2}}{2 \sigma^{2}(a, n+1)}\right\}+\varepsilon(a, n+1 ; T)
$$

where $\mu(a, n+1)$ and $\sigma^{2}(a, n+1)$ are the mean and the variance of $T$ respectively (see Appendix A);

$$
\mu(a, n+1)=\sum_{j=a}^{n} \frac{1}{j^{\alpha}} ; \quad \sigma^{2}(a, n+1)=\sum_{j=a}^{n} \frac{1}{j^{2 \alpha}} .
$$

We note for future reference that $\sigma^{2}(a, n+1) \rightarrow \infty$ as $n \rightarrow \infty$ when $\alpha \leq \frac{1}{2}$. The function $\varepsilon(a, n+1 ; T)$, which describes the deviation from normality, is bounded in the strict sense that (see Appendix B),

$$
R(a, n+1) \equiv \sup _{T \in \Re}\left|\int_{-\infty}^{T} \varepsilon\left(a, n+1 ; T^{\prime}\right) d T^{\prime}\right| \leq \frac{C^{\prime}}{\left[\sigma^{2}(a, n+1)\right]^{3 / 2}} \sum_{j=a}^{n} \frac{1}{j^{3 \alpha}}
$$

where $C^{\prime}$ is a constant of order unity. One can now employ (7) in conjunction with (6) to derive accurate approximations for the exit probability in various limits. First we extend the lower limit of both integrals in (6) to $-\infty$, which introduces no error since the probability density is zero for $T<0$. After carrying out the 'leading order' double integral one obtains, 


$$
E_{n}(a, b)=2 \Phi\left[\frac{\mu(a, n+1)-\mu(b, n+1)}{\sqrt{\sigma^{2}(a, n+1)+\sigma^{2}(b, n+1)}}\right]+\delta E_{n}(a, b)
$$

where $\Phi(z)$ with $z \leq 0$ is simply the cumulative distribution function of the standard normal distribution (which in turn may be written in terms of the error function);

$$
\Phi(z) \equiv \frac{1}{\sqrt{2 \pi}} \int_{-\infty}^{z} e^{-x^{2} / 2} d x=\frac{1}{2}+\frac{1}{2} \operatorname{erf}\left(\frac{\mathrm{z}}{\sqrt{2}}\right)
$$

and use has been made of the integral identity,

$$
\frac{1}{2 \pi} \int_{-\infty}^{\infty} e^{-x^{2} / 2} \int_{-\infty}^{u x+r} e^{-y^{2} / 2} d y d x \equiv \Phi\left[\frac{r}{\sqrt{1+u^{2}}}\right]
$$

The 'correction' term $\delta E_{n}(a, b)$ in (10) contains the contributions to (6) arising from integrals involving the functions $\varepsilon(a, n+1 ; T)$ and $\varepsilon(b, n+1 ; T)$. Elementary manipulations show that $\delta E_{n}(a, a)=0$; more generally, using the definition in (9) one has the bound $\left|\delta E_{n}(a, b)\right|<2 R(a, n+1)+2 R(b, n+1)$. When $\alpha \leq \frac{1}{2}$ it follows from $(8,9)$ that $R(a, n+1)$ and $R(b, n+1)$ must tend to zero as $n \rightarrow \infty$ for any fixed $a, b$ and so $\delta E_{n}(a, b) \rightarrow 0$ as $n \rightarrow \infty$. Moreover, since the argument of the first term in (10) tends to zero from below as $n \rightarrow \infty$, this rigorously proves that $E_{n}(a, b) \rightarrow 1$ as $n \rightarrow \infty$ for $\alpha \leq \frac{1}{2}$. When $\alpha>\frac{1}{2}$ the situation is different since one cannot deduce from $(8,9)$ that $R(a, n+1)$ and $R(b, n+1)$ tend to zero as $n \rightarrow \infty$ for fixed $a, b$; 
indeed they are strictly positive in the limit. However, if in addition we let $a, b \rightarrow \infty$ in such a way that $n-a \rightarrow \infty$ and $n-b \rightarrow \infty$ also, then once again one may show using $(8,9)$ that $R(a, n+1)$ and $R(b, n+1)$ must tend to zero and so $\delta E_{n}(a, b) \rightarrow 0$. Under these conditions the first term in (10) provides a progressively more accurate estimate for $E_{n}(a, b)$ for any value of $\alpha$. Numerically this term is found to provide an excellent approximation to $E_{n}(a, b)$ if $a$ and $b$ are close, even if $a$ and $b$ are relatively small.

We can refine this argument and expand upon what is meant by 'close' by defining the scaling variables $N, \rho$ and $\kappa$ such that,

$$
N=a+b, \quad \rho=\frac{a-b}{\sqrt{a+b}}, \quad \kappa=\frac{2 n}{a+b}
$$

and then considering the behaviour of $E_{n}(a, b)$ in the limit $N \rightarrow \infty$ with $\rho>0$ and $\kappa>1$ fixed but otherwise arbitrary. Rearranging (13) one sees that $a=\frac{1}{2}[N+\rho \sqrt{N}]$, $b=\frac{1}{2}[N-\rho \sqrt{N}]$ and $n=\frac{1}{2} \kappa N$; thus these primary variables tend to infinity in such a way that $\delta E_{n}(a, b) \rightarrow 0$ for any value of $\alpha$. It follows that,

$$
\lim _{N \rightarrow \infty} E(N, \rho, \kappa)=2 \Phi\left[-\rho \frac{|1-2 \alpha|^{1 / 2}}{\left|\kappa^{1-2 \alpha}-1\right|^{1 / 2}}\right]
$$

and this is exact in the sense that the correction terms vanish as $N \rightarrow \infty$ for all $\alpha$. The result $E_{\infty}^{(\alpha=1)}(a, b)=2 \Phi[-\rho]+O\left(N^{-1}\right)$ derived in Appendix C for the case $n=\infty$, 
$\alpha=1$ supports the argument that, for fixed $\rho$ and $\kappa,(14)$ provides a progressively better approximation the larger the value of $N=a+b$. Written explicitly in terms of the primary variables (now assumed to be finite but large) one therefore has the asymptotically precise estimate $\hat{E}_{n}(a, b)=2 \Phi\left[-\theta_{n}(a, b)\right]$ where

$$
\theta_{n}(a, b)=\frac{(a-b)}{\sqrt{a+b}} \times \begin{cases}\frac{(1-2 \alpha)^{1 / 2}}{\left[\left(\frac{2 n}{a+b}\right)^{1-2 \alpha}-1\right]^{1 / 2}} & \alpha<\frac{1}{2} \\ \frac{(2 \alpha-1)^{1 / 2}}{\left[1-\left(\frac{a+b}{2 n}\right)^{2 \alpha-1}\right]^{1 / 2}} & \alpha>\frac{1}{2} .\end{cases}
$$

This is consistent with the fact that $E_{\infty}(a, b)=1$ if $\alpha<\frac{1}{2}$ whilst $E_{\infty}(a, b)<1$ if $\alpha>\frac{1}{2}$. Thus $\alpha=\frac{1}{2}$ marks a point of transition and we have an explicit estimate when $\alpha>\frac{1}{2}$ for the limiting value $\hat{E}_{\infty}(a, b)=2 \Phi[-\rho \sqrt{2 \alpha-1}]$. This generalizes the result given above for $\alpha=1$ and is more accurate the smaller the value of $\rho$ (which then provides a measure of $a$ and $b$ being close). At the critical point $\alpha=\frac{1}{2}$ itself one can infer from (15) in a limiting sense;

$$
\theta_{n}^{\left(\alpha=\frac{1}{2}\right)}(a, b)=\frac{(a-b)}{\sqrt{a+b}}\left[\log \left(\frac{2 n}{a+b}\right)\right]^{-1 / 2}
$$


which tends to zero (albeit slowly) as $n \rightarrow \infty$. This is also consistent with the fact that the critical case $\alpha=\frac{1}{2}$ is marginally recurrent, i.e. $E_{\infty}^{\left(\alpha=\frac{1}{2}\right)}(a, b)=1$. One can use $(15,16)$ to accurately describe the crossover behaviour over a broad parameter range.

\subsection{The survival probability}

The survival probability $\bar{E}_{n}(a, b) \equiv 1-E_{n}(a, b)$ is the probability that a given path which starts at $(a, b)$ does not reach the line $B=A$ by the point $(n, n)$, i.e. rolereversal does not occur within the first $S=2 n-a-b$ steps. In the case where $\alpha \leq \frac{1}{2}$ we have $E_{n}(a, b) \rightarrow 1$ as $n \rightarrow \infty$ so the survival probability vanishes and from the way in which it vanishes one can define a persistence exponent $\theta_{p}$. Using $(15,16)$

one may infer when $\alpha<\frac{1}{2}$ that $\bar{E}_{n}(a, b)=O\left(n^{-(1 / 2-\alpha)}\right)$ as $n \rightarrow \infty$, so that $\theta_{p}=\frac{1}{2}-\alpha$. This reproduces and extends the known result when $\alpha=0$ that $\theta_{p}=\frac{1}{2}$ for an isotropic random walk $[18,19]$. More specifically as $n \rightarrow \infty$ for values of $a$ and $b$ which are sufficiently large and close,

$$
\bar{E}_{n}(a, b) \approx \sqrt{\frac{2}{\pi}} \frac{(a-b)}{\sqrt{a+b}} \times \begin{cases}(1-2 \alpha)^{1 / 2}\left(\frac{a+b}{2}\right)^{1 / 2-\alpha} \frac{1}{n^{1 / 2-\alpha}} & \alpha<\frac{1}{2} \\ {\left[\log \left(\frac{2 n}{a+b}\right)\right]^{-1 / 2}} & \alpha=\frac{1}{2} .\end{cases}
$$


In figure 4 the relevant data from figure 3 is re-plotted and comparison is made with the asymptotic estimates provided by (17). The agreement for large $n$ is seen to be good, even for the marginal case $\alpha=\frac{1}{2}$.

Interestingly, essentially the same results are found for the survival probability of a particular form of history-dependent random walk [23, 24]. To understand why this is the case, and building on the observations made in [25], consider again the random walk associated with the population difference variable $\Delta \equiv A-B$ as a function of the number of steps $S$. One can write $A=\frac{1}{2}(S+a+b)+\frac{1}{2} \Delta$ and $B=\frac{1}{2}(S+a+b)-\frac{1}{2} \Delta$, whereupon, as long as $\Delta=o(S)$ as $S \rightarrow \infty$, based on (1) one has for the transition probabilities at a given step after a large number of steps,

$$
\operatorname{Pr}(\Delta \rightarrow \Delta \pm 1) \approx \frac{1}{2}\left[1 \pm \alpha\left(\frac{\Delta}{S+a+b}\right)\right]
$$

This is, in essence, the model studied in [23, 24]. The structure of (18) implies longrange correlations whose strength is controlled by the parameter $\alpha$. The analysis presented in $[23,24]$ shows (based on a corresponding continuous-time FokkerPlanck equation) that the dynamics under (18) become super-diffusive when $\alpha>\frac{1}{2}$; more precisely, the standard deviation of the process $\sigma_{S}=O\left(S^{1 / 2}\right)$ when $\alpha<\frac{1}{2}$ and $\sigma_{S}=O\left(S^{\alpha}\right)$ when $\frac{1}{2}<\alpha<1$. In the current context this corresponds to the change from recurrent to transient behaviour evident in the exit probability $E_{n}(a, b)$. 


\subsection{The role-reversal probability}

There are situations where one is directly interested in the probability $P_{S}(B>A)$ that after $S$ steps role-reversal has occurred. There is a simple but important relationship between $P_{S}(B>A)$ and $E_{n}(a, b)$ which may be derived as follows. Suppose $S+a+b$ is odd and consider the line $A+B=2 n+1$ which will be reached in $S$ steps where $S=2 n-a-b+1$; see figure 1 . Of all the lattice paths which contribute to $E_{n}(a, b)$, i.e. that reach the line $B=A$ at some point $(k, k)$ where $k \leq n$, precisely half (carrying half the probabilistic weight by symmetry) will subsequently intersect the line $A+B=2 n+1$ at points where $B>A$ (see figure 1). It follows immediately that $P_{S}(B>A)=\frac{1}{2} E_{n}(a, b)$ where $n=\left\lfloor\frac{1}{2}(S+a+b)\right\rfloor$ and the results in Section 3.2 may therefore be translated into a different context. This is a key result. In particular, in the limit $S \rightarrow \infty$ we have that $P_{\infty}(B>A)=\frac{1}{2} E_{\infty}(a, b)$. This latter result was given in [33] for the standard Pólya urn, i.e. for $\alpha=1$ only; the result here is of much greater generality. We note in particular that $P_{\infty}(B>A)=\frac{1}{2}$ if $\alpha \leq \frac{1}{2}$ and so in this regime the long-time behaviour is insensitive to the initial condition.

If $S+a+b$ is even rather than odd the situation is slightly more complicated. By now considering the line $A+B=2 n$ which will be reached in $S$ steps where $S=2 n-a-b$ one can show that $P_{S}(B>A)=\frac{1}{2} E_{n}(a, b)-\frac{1}{2} P_{S}(B=A)$ where $n=\frac{1}{2}(S+a+b)$. The correction term $\frac{1}{2} P_{S}(B=A)$ arises due to the fact that the point $(n, n)$ lies on the line $A+B=2 n$ when $S+a+b$ is even. Although we do not have an explicit expression for $P_{S}(B=A)$, it is clear it becomes ever smaller as $S \rightarrow \infty$ 
and vanishes in the limit. Since $P_{S}(B=A)$ is zero if $S+a+b$ is odd, for completeness one can write quite generally $P_{S}(B>A)=\frac{1}{2} E_{n}(a, b)-\frac{1}{2} P_{S}(B=A)$ where $n=\left\lfloor\frac{1}{2}(S+a+b)\right\rfloor$ and this holds for all $S \geq a-b$.

\subsection{The first passage probability}

Turning attention to the first passage probability $G_{n}(a, b)$ itself, this can be obtained from (4) using the fact that $G_{n}(a, b)=E_{n}(a, b)-E_{n-1}(a, b)$;

$$
G_{n}(a, b)=\frac{2}{n^{2 \alpha}} \sum_{j=a}^{n} j^{2 \alpha}\left\{\prod_{\substack{\ell=a \\ \ell \neq j}}^{n} \frac{\ell^{\alpha}}{\ell^{\alpha}-j^{\alpha}}\right\}\left\{\prod_{\ell^{\prime}=b}^{n} \frac{\ell^{\prime \alpha}}{\ell^{\prime \alpha}+j^{\alpha}}\right\} ; \quad \alpha \neq 0 .
$$

This satisfies $G_{n}(a, a)=0$ for all $n>a$ with $G_{a}(a, a) \equiv 1$. In general, the rate of decay as $n \rightarrow \infty$ is of most interest and based on the analysis that led to $(15,16)$ one finds for values of $a$ and $b$ which are sufficiently large and close,

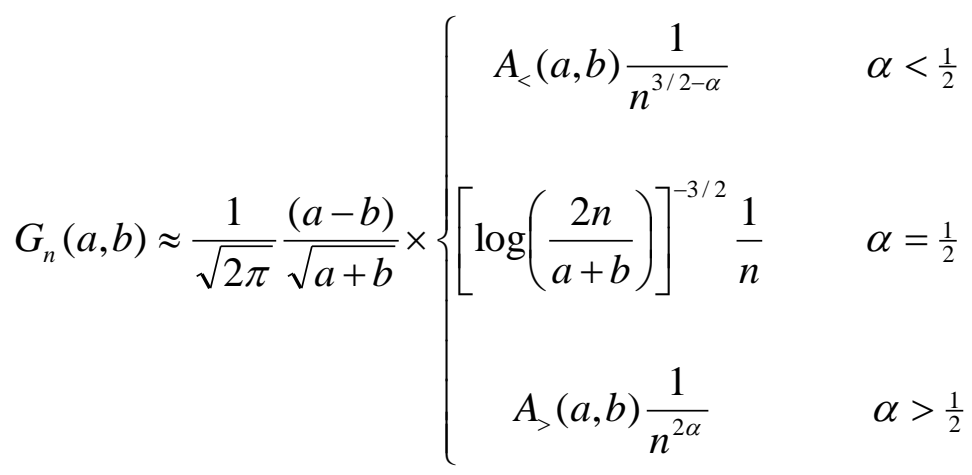

with amplitudes, 


$$
\begin{aligned}
& A_{<}(a, b)=(1-2 \alpha)^{3 / 2}\left(\frac{a+b}{2}\right)^{1 / 2-\alpha} \\
& A_{>}(a, b)=(2 \alpha-1)^{3 / 2}\left(\frac{a+b}{2}\right)^{2 \alpha-1} \exp \left(-\frac{(2 \alpha-1)(a-b)^{2}}{2(a+b)}\right) .
\end{aligned}
$$

In figure 5 we compare the results for $G_{n}(a, b)$ based on simulations with the results derived from the estimates given by $(20,21)$. The agreement for large $n$ is very good, even for the critical case $\alpha=\frac{1}{2}$ when the convergence is slowest since the rate of decay of $G_{n}(a, b)$ is slowest. One can see based on (20) that, although role-reversal will happen with probability one for $\alpha \leq \frac{1}{2}$, the expected number of steps or time for this to happen is infinite if $-\frac{1}{2} \leq \alpha \leq \frac{1}{2}$, and only becomes finite if $\alpha<-\frac{1}{2}$. Such an insight is beyond that which can be deduced from the long-time asymptotic dynamics (as discussed in the Introduction) alone.

\subsection{Special cases}

For the special case $\alpha=0$ there is a simple combinatorial derivation invoking the reflection principle [32] and adapting the approach taken in Appendix C. One obtains,

$$
\begin{aligned}
& E_{n}^{(\alpha=0)}(a, b)=(a-b) \sum_{j=a}^{n} \frac{(2 j-a-b-1) !}{(j-a) !(j-b) !}\left(\frac{1}{2}\right)^{2 j-a-b} \\
& G_{n}^{(\alpha=0)}(a, b)=(a-b) \frac{(2 n-a-b-1) !}{(n-a) !(n-b) !}\left(\frac{1}{2}\right)^{2 n-a-b} .
\end{aligned}
$$


The expression for the limiting exit probability as $n \rightarrow \infty$ coincides with a particular hypergeometric function with its primary argument set equal to unity. Using Gauss' hypergeometric theorem and other algebraic manipulations it may then be confirmed that $E_{\infty}^{(\alpha=0)}(a, b)=1$, irrespective of the starting point. The behaviour as $n \rightarrow \infty$ deduced from (23) using Stirling's approximation agrees precisely with (20).

From (4) and (19) we obtain for the case $\alpha=1$, which corresponds to the standard Pólya urn model,

$$
\begin{aligned}
& E_{n}^{(\alpha=1)}(a, b)=\frac{2 n !^{2}}{(a-1) !(b-1) !} \sum_{j=a}^{n} \frac{(-1)^{j-a}(j+b-1) !}{j(j-a) !(n-j) !(n+j) !} \\
& G_{n}^{(\alpha=1)}(a, b)=\frac{2(n-1) !^{2}}{(a-1) !(b-1) !} \sum_{j=a}^{n} \frac{(-1)^{j-a} j(j+b-1) !}{(j-a) !(n-j) !(n+j) !} .
\end{aligned}
$$

These expressions are quite different in appearance from the expressions given in Appendix C which follow from conventional combinatorial arguments [17], but they may be shown to be equivalent numerically. It would be an interesting challenge to establish the equivalence by direct algebraic manipulation, but we do not pursue that here. Likewise one can derive the same quantities for the case $\alpha=-1$, which corresponds to the Friedman urn model for which there is no simple combinatorial derivation;

$$
E_{n}^{(\alpha=-1)}(a, b)=2 \sum_{j=a}^{n} \frac{(-1)^{n-j} j^{2 n-a-b+1}(j+b-1) !}{(j-a) !(n-j) !(n+j) !}
$$




$$
G_{n}^{(\alpha=-1)}(a, b)=2 n^{2} \sum_{j=a}^{n} \frac{(-1)^{n-j} j^{2 n-a-b-1}(j+b-1) !}{(j-a) !(n-j) !(n+j) !} .
$$

Calculating the first passage properties of the Friedman urn was posed as a challenge in [17], where it was conjectured that these would be much more analogous to the isotropic random walk than the standard Pólya urn. The wider analysis above encapsulated in (15) and (20) shows that this is indeed the case.

\section{Discussion}

In this paper we have considered the first passage properties of a generalized twocomponent Pólya urn process, deriving exact results for the exit probability (4) and the related first passage probability (19). These results are not especially intuitive in isolation and their computation requires high numerical precision when the arguments are large. However, the integral representation (6) provides the basis for a powerful scheme for making asymptotically accurate approximations which capture the richness of the model both qualitatively and quantitatively. This complements previous analyses and also offers new insights into the behavioural change which occurs at the defined parameter value $\alpha=\frac{1}{2}$.

From the perspective of population growth the parameter $\alpha$ provides a measure of the extent to which the elements of the populations are equally 'participative' in determining their subsequent evolution. Related ideas may be found in recent studies of 'cumulative advantage' in growing networks [16]. A very simple model of physical 
aggregation in $d$-dimensions illustrates the concept and its subtlety. Imagine randomly arriving particles adhere to one of two spherical clusters, and suppose the probability of adhering is simply determined by relative volume. Then (1) applies with $\alpha=1$; this is the standard Pólya urn. If, on the other hand, the probability of adhering is determined by relative surface area, then (1) applies with $\alpha=1-d^{-1}$. One might ask in this latter case whether, given an initial size imbalance, role-reversal is guaranteed to occur, i.e. that the two clusters are guaranteed to become equal in volume at some point in time if one waits long enough. The results here demonstrate that in three dimensions the answer is no $\left(\alpha=\frac{2}{3}\right)$, whilst in two dimensions the answer is yes, but only just ( $\alpha=\frac{1}{2}$, the marginal case). The results also quantify the behaviour in several important respects.

Various extensions of the work presented suggest themselves. One idea, given the generality of the embedding approach, would be to replace the 'urn function' $x^{\alpha}$ in (1) with another function $\lambda(x)$. It is worth noting that the method of derivation is general enough to show that (4) and (19) remain valid for any non-degenerate set of variables $\{\lambda(k)\}$ under the universal replacement $k^{\alpha} \rightarrow \lambda(k)$. One could also define more general urn functions $\lambda(x)$ and $\mu(y)$ which would allow for different treatment of each of the two populations. This may be directly applicable to the models discussed in [16]. From the perspective of history-dependent random walks, having noted the connection with the previous work discussed in $[23,24]$, it would be interesting to explore this link in more detail and perhaps also relate the present work to other types of random walks with memory [34-37]. Another possible avenue to explore is the recent study of random walks with extreme value memory discussed in 
[38]. Finally, we note a strong parallel with ideas emerging from studies of other generalised Pólya urn processes based on a scaling analysis of the correlation function relating the first and last step [39].

\section{Acknowledgements}

The authors would like to thank Philip Aston and Andrew Pye for support with regard to the simulations and numerical results.

\section{Appendix A. Birth processes}

Let $X(t)$ be a birth process on the non-negative integers $j$ with state-dependent transition rates $j^{\alpha}$ and initial condition $X(0)=a$, and suppose there is an absorbing state at $j=n+1$ where $n \geq a$. The probability $P(a, k ; t)$ of being in state $k>a$ at time $t$ satisfies the master equation,

$$
\begin{aligned}
& \frac{d}{d t} P(a, k ; t)=(k-1)^{\alpha} P(a, k-1 ; t)-k^{\alpha} P(a, k ; t) ; \quad k \leq n \\
& \frac{d}{d t} P(a, n+1 ; t)=k^{\alpha} P(a, n ; t)
\end{aligned}
$$

with $P(a, a ; t)=e^{-a^{\alpha} t}$. Let $\tilde{P}(a, k ; s)$ be the Laplace transform of $P(a, k ; t)$. From (A1) one obtains a recursion in the variable $k$ which may be iterated to give, 


$$
\begin{aligned}
& \tilde{P}(a, k ; s)=\frac{1}{k^{\alpha}} \prod_{j=a}^{k} \frac{j^{\alpha}}{j^{\alpha}+s} ; \quad k \leq n \\
& \tilde{P}(a, n+1 ; s)=\frac{1}{s} \prod_{j=a}^{n} \frac{j^{\alpha}}{j^{\alpha}+s} .
\end{aligned}
$$

The inversion is an elementary exercise in residue calculus and one obtains from the first result in (A2) for $k \leq n$,

$$
P(a, k ; t)=\frac{1}{k^{\alpha}} \sum_{j=a}^{k} j^{\alpha}\left\{\prod_{\substack{\ell=a \\ \ell \neq j}}^{k} \frac{\ell^{\alpha}}{\ell^{\alpha}-j^{\alpha}}\right\} e^{-j^{\alpha} t} ; \quad \alpha \neq 0 .
$$

For the absorbing state $j=n+1$ the inversion of the second result in (A2) gives,

$$
P(a, n+1 ; t)=1-\sum_{j=a}^{n}\left\{\prod_{\substack{\ell=a \\ \ell \neq j}}^{n} \frac{\ell^{\alpha}}{\ell^{\alpha}-j^{\alpha}}\right\} e^{-j^{\alpha} t} ; \quad \alpha \neq 0 .
$$

One observes that $P(a, n+1 ; t) \rightarrow 1$ as $t \rightarrow \infty$, since every realisation of $X(t)$ will eventually be absorbed.

The probability density function $p(a, k ; T)$ for the first passage time $T$ to reach state $k>a$ is simply the transition rate from state $k-1$ multiplied by the probability of being in state $k-1$ at time $T$. In other words, $p(a, k ; T)=(k-1)^{\alpha} P(a, k-1 ; T)$. Using (A2) and (A3) one obtains for $k \leq n+1$, 


$$
\begin{aligned}
& \tilde{p}(a, k ; s)=\prod_{j=a}^{k-1} \frac{j^{\alpha}}{j^{\alpha}+s} \\
& p(a, k ; T)=\sum_{j=a}^{k-1} j^{\alpha}\left\{\prod_{\substack{\ell=a \\
\ell \neq j}}^{k-1} \frac{\ell^{\alpha}}{\ell^{\alpha}-j^{\alpha}}\right\} e^{-j^{\alpha} T} ; \quad \alpha \neq 0 .
\end{aligned}
$$

Since $\tilde{p}(a, k ; s=0)=1$ it follows that $\int_{0}^{\infty} p(a, k ; T) d T=1$, as must be the case because all states are eventually accessed. Further, since the state $k=n+1$ is absorbing one must have $P(a, n+1 ; t)=\int_{0}^{t} p(a, n+1 ; T) d T$. To derive the mean $\mu(a, k)$ and variance $\sigma^{2}(a, k)$ of $T$ one simply has to note that the time $T_{j}$ spent in state $j$ has density function $p\left(j, j+1, T_{j}\right)=j^{\alpha} e^{-j^{\alpha} T_{j}}$, mean value $\mu_{j}=j^{-\alpha}$ and variance $\sigma_{j}^{2}=j^{-2 \alpha}$. Given that the first passage time $T=\sum_{j=a}^{k-1} T_{j}$, it follows immediately that,

$$
\mu(a, k)=\sum_{j=a}^{k-1} \frac{1}{j^{\alpha}} ; \quad \sigma^{2}(a, k)=\sum_{j=a}^{k-1} \frac{1}{j^{2 \alpha}} .
$$

By retaking the Laplace transform of (A6) and equating it to (A5) one obtains a partial fraction identity which is true for all $s$;

$$
\sum_{j=a}^{k-1}\left\{\prod_{\substack{\ell=a \\ \ell \neq j}}^{k-1} \frac{\ell^{\alpha}}{\ell^{\alpha}-j^{\alpha}}\right\} \frac{j^{\alpha}}{j^{\alpha}+s} \equiv \prod_{j=a}^{k-1} \frac{j^{\alpha}}{j^{\alpha}+s} ; \quad \alpha \neq 0 .
$$


By setting $s=0$ one can show in conjunction with (A4) that $P(a, n+1 ; t=0)=0$, as must be the case given the initial condition. Also, by taking the limit $s \rightarrow \infty$ one can show from (A6) that $p(a, k ; T=0)=0$ for $k>a+1$.

\section{Appendix B. Asymptotic normality}

Under fairly general limiting conditions where $k, a \rightarrow \infty$ in such a way that $k-a \rightarrow \infty$ as well, the first passage time $T$ to reach state $k$ starting from state $a$ is asymptotically normal in the sense that,

$$
Z \equiv \frac{T-\mu(a, k)}{\sigma(a, k)} \stackrel{D}{\rightarrow} N(0,1)
$$

where $N(0,1)$ denotes standard normal distribution (zero mean, unit variance) whose cumulative distribution function $\Phi(z)$ is given by,

$$
\Phi(z) \equiv \frac{1}{\sqrt{2 \pi}} \int_{-\infty}^{z} e^{-x^{2} / 2} d x
$$

The proof follows from the central limit theorem for independent but non-identically distributed random variables; these variables are the mean-shifted state times $T_{j}-\mu_{j}$, where $T-\mu(a, k)=\Sigma_{j=a}^{k-1}\left(T_{j}-\mu_{j}\right)$. Application of the Berry-Esseen theorem [32] implies that the cumulative distribution $F(a, k, z)$ of the variable $Z$ satisfies, 


$$
\sup _{z \in \mathcal{R}}|F(a, k, z)-\Phi(z)| \leq \frac{C}{\left[\sigma^{2}(a, k)\right]^{3 / 2}} \sum_{j=a}^{k-1} \mathrm{E}\left[\left|T_{j}-\mu_{j}\right|^{3}\right]
$$

for some constant $C$ (which is less than unity). The expectation $\mathrm{E}\left[\left|T_{j}-\mu_{j}\right|^{3}\right]$ may be calculated explicitly using results from Appendix A;

$$
\mathrm{E}\left[\left|T_{j}-\mu_{j}\right|^{3}\right]=\frac{12 e^{-1}-2}{j^{3 \alpha}}=\frac{2.414 \ldots}{j^{3 \alpha}} .
$$

Under the stated limiting conditions $k, a \rightarrow \infty$ with $k-a \rightarrow \infty$ the right hand side of (B3) tends to zero for all $\alpha$, which establishes (B1).

Alternatively, the characteristic function $\phi_{z}(a, k ; \omega)$ of the variable $Z$ may be obtained from the Laplace transform (A5) after a change of variables;

$$
\phi_{Z}(a, k ; \omega)=e^{-i \omega \mu(a, k) / \sigma(a, k)} \prod_{j=a}^{k-1}\left(1-\frac{i \omega}{j^{\alpha} \sigma(a, k)}\right)^{-1}
$$

which after taking logarithms may be written as,

$$
\log \phi_{Z}(a, k ; \omega)=-i \omega \frac{\mu(a, k)}{\sigma(a, k)}-\sum_{j=a}^{k-1} \log \left(1-\frac{i \omega}{j^{\alpha} \sigma(a, k)}\right)
$$

In the stated limit one may expand the logarithm on the right hand side of (B6) to derive, 


$$
\log \phi_{Z}(a, k ; \omega)=-\frac{\omega^{2}}{2}+\frac{i \omega^{3}}{3\left[\sigma^{2}(a, k)\right]^{3 / 2}} \sum_{j=a}^{k-1} \frac{1}{j^{3 \alpha}}+\ldots \rightarrow-\frac{\omega^{2}}{2}
$$

and this also establishes (B1) since $e^{-\omega^{2} / 2}$ is the characteristic function of the standard normal distribution density $\frac{1}{\sqrt{2 \pi}} e^{-z^{2} / 2}[32]$.

\section{Appendix C. The standard Pólya urn}

When $\alpha=1$ the probabilistic weight of each contributing path based on (1) between start point $(a, b)$ and end point $(A, B)$ is the same. The end-point probability $P_{S}^{(\alpha=1)}(A, B)$ that, after a fixed number of steps $S$ a path finishes at the point $(A, B)$, where $S=A+B-a-b$, is then given by,

$$
P_{S}^{(\alpha=1)}(A, B)=\frac{(A+B-a-b) !}{(A-a) !(B-b) !} \times \frac{(a+b-1) !(A-1) !(B-1) !}{(a-1) !(b-1) !(A+B-1) !} .
$$

Using (C1) and by invoking the reflection principle, the first passage probability that a path reaches the line $B=A$ for the first time at the point $(n, n)$ is [17],

$$
\begin{aligned}
G_{n}^{(\alpha=1)}(a, b) & =\frac{a-b}{2 n-a-b} \times P_{2 n-a-b}^{(\alpha=1)}(n, n) \\
& =\frac{(a-b)(a+b-1) !(2 n-a-b-1) !(n-1) !^{2}}{(a-1) !(b-1) !(n-a) !(n-b) !(2 n-1) !} .
\end{aligned}
$$


From this one can write down an expression for the exit probability that a path of the process reaches the line $B=A$ no later than the point $(n, n)$ using the defining relation $E_{n}(a, b) \equiv \sum_{j=a}^{n} G_{j}(a, b)$;

$$
E_{n}^{(\alpha=1)}(a, b)=\frac{(a-b)(a+b-1) !}{(a-1) !(b-1) !} \sum_{j=a}^{n} \frac{(2 j-a-b-1) !(j-1) !^{2}}{(j-a) !(j-b) !(2 j-1) !}
$$

As shown in [17], using Gauss' hypergeometric theorem and other algebraic manipulations one may derive from (C3) various non-trivial results regarding the limiting probability $E_{\infty}^{(\alpha=1)}(a, b)$. Here we note another way to obtain the same results.

The probability $P_{S}^{(\alpha=1)}(A, B)$ given by $(\mathrm{C} 1)$ may be analysed using Stirling's approximation to derive as $S \rightarrow \infty$ with $A=O(S)$ and $B=O(S)$,

$$
P_{S}^{(\alpha=1)}(A, B)=\frac{(a+b-1) !}{(a-1) !(b-1) !}\left(\frac{A-a}{S}\right)^{a-1}\left(\frac{B-b}{S}\right)^{b-1} \frac{1}{S}\left[1+O\left(\frac{1}{S}\right)\right] .
$$

This two-parameter beta distribution is a well-known result [3, 4]. From it one can calculate the probability that role-reversal has occurred $P_{S}^{(\alpha=1)}(B>A)$ by summing over points for which $A<\frac{1}{2}(S+a+b)$. By exploiting the general relationship $P_{\infty}(B>A)=\frac{1}{2} E_{\infty}(a, b)$ derived in the main text one has [33],

$$
E_{\infty}^{(\alpha=1)}(a, b)=2 P_{\infty}^{(\alpha=1)}(B>A)=\frac{2(a+b-1) !}{(a-1) !(b-1) !} \int_{0}^{1 / 2} x^{a-1}(1-x)^{b-1} d x .
$$


From (C5) one can confirm that $E_{\infty}^{(\alpha=1)}(a, a)=1$, as well as quickly derive results for particular cases such as $E_{\infty}^{(\alpha=1)}(a, 1)=2^{1-a}, E_{\infty}^{(\alpha=1)}(a, 2)=(a+2) 2^{-a}$ etc. More importantly, one may show that $E_{\infty}^{(\alpha=1)}(a, b)<1$ for all $a>b$. This means that a given path is not guaranteed to reach the line $B=A$, in other words, the process is transient.

The asymptotic behaviour of $E_{\infty}^{(\alpha=1)}(a, b)$ when $a$ and $b$ are large can be derived from (C5). Define the scaling variables $N$ and $\rho$ such that,

$$
N=a+b, \quad \rho=\frac{a-b}{\sqrt{a+b}}
$$

and consider the behaviour of $E_{\infty}^{(\alpha=1)}(a, b)$ in the limit $N \rightarrow \infty$ with $\rho>0$ fixed.

Noting that $a=\frac{1}{2}[N+\rho \sqrt{N}]$ and $b=\frac{1}{2}[N-\rho \sqrt{N}]$ one may show by expanding the integrand of (C5) around the upper limit, together with the use of Stirling's approximation, that,

$$
\begin{aligned}
E_{\infty}^{(\alpha=1)}(a, b) & =1-\sqrt{\frac{2}{\pi}} \int_{-\rho}^{0} e^{-x^{2} / 2} d x+O\left(\frac{1}{N}\right) \\
& =2 \Phi[-\rho]+O\left(\frac{1}{N}\right) .
\end{aligned}
$$

The leading term of (C7) was proposed in [17] on the basis of heuristic arguments; the derivation here is mathematically precise and the error term is tight [7]. 


\section{References}

[1] Eggenberger F and Pólya G 1923 Zeit. Angew. Math. Mech. 3279

[2] Pólya G 1931 Ann. Inst. H. Poincare 1117

[3] Johnson N L and Kotz S 1977 Urn Models and Their Application: An Approach to Modern Discrete Probability Theory (New York: Wiley)

[4] Mahmoud H M 2009 Pólya urn models (Boca Raton, FL: CRC Press)

[5] Friedman B 1949 Commun. Pure Appl. Math. 259

[6] Freedman D 1965 Ann. Math. Stat. 36956

[7] Flajolet P, Dumas P and Puyhaubert V 2006 Colloquium on Mathematics and Computer Science, Discrete Mathematics and Theoretical Computer Science Proc. AG (DMTCS Nancy, France) pp 59-118

[8] Kingman J F C and Volkov S E 2003 J. Theor. Probab. 16267

[9] Wei L J 1979 Ann. Stat. 7291

[10] Khanin K and Khanin R 2001 J. Math. Biol. 4226

[11] Barabasi A -L and Albert R 1999 Science 286509

[12] Krapivsky P L and Redner S 2001 Phys. Rev. E 63066123

[13] Oliveira R and Spencer J 2005 Internet Math. 2121

[14] Arthur W B, Ermoliev Yu M and Kaniovski Yu M 1987 Eur. J. Oper. Res. 30 294

[15] Arthur W B 1989 The Economic Journal 99116

[16] Jiang B, Sun L, Figueiredo D R, Ribeiro B and Towsley D 2015 J. Stat. Mech.: Theor. Expt. P11022

[17] Antal T, Ben-Naim E and Krapivsky P L 2010 J. Stat. Mech.: Theor. Expt. P07009 
[18] Redner S 2001 A Guide to First Passage Processes (Cambridge: Cambridge University Press)

[19] Bray A J, Majumdar S N and Schehr G 2103 Adv. Phys. 62225

[20] Oliveira R and Spencer J 2005 arXiv preprint math/0510663

[21] Oliveira R 2008 Combin. Prob. Comp. 1787

[22] Oliveira R 2009 Rand. Struct. Algorithms 34454

[23] Hod S and Keshet U 2004 Phys. Rev. E 70 015104(R)

[24] Keshet U and Hod S 2005 Phys. Rev. E 72046144

[25] Huillet T 2008 J. Phys. A: Math. Theor. 41505005

[26] Harris T E 1989 The Theory of Branching Processes (New York: Dover)

[27] Athreya K B and Karlin S 1968 Ann. Math. Stat. 391801

[28] Hill B M, Lane D and Sudderth W 1980 Ann. Probab. 8214

[29] Davis B 1990 Prob.Theor. Rel. Fields 84203

[30] Janson S 2004 Stochastic Processes and their Applications 110177

[31] Pemantle R 2007 Probability Surveys 41

[32] Feller W 1971 An Introduction to Probability Theory and its Applications (New York: Wiley)

[33] Wallstrom T C 2012 Amer. Math. Month. 119516

[34] Usatenko O V and Yampol'skii V A 2003 Phys. Rev. Lett. 90110601

[35] Schutz G M and Trimper S 2004 Phys. Rev. E 70045101

[36] Paraan F N C and Esguerra J P 2006 Phys. Rev. E 74032101

[37] Hisakado M and Mori S 2010 J. Phys. A: Math. Theor. 43315207

[38] Harris R J 2015 New J. Phys. 17053049

[39] Mori S and Hisakado M 2015 Phys. Rev. E 92052112 


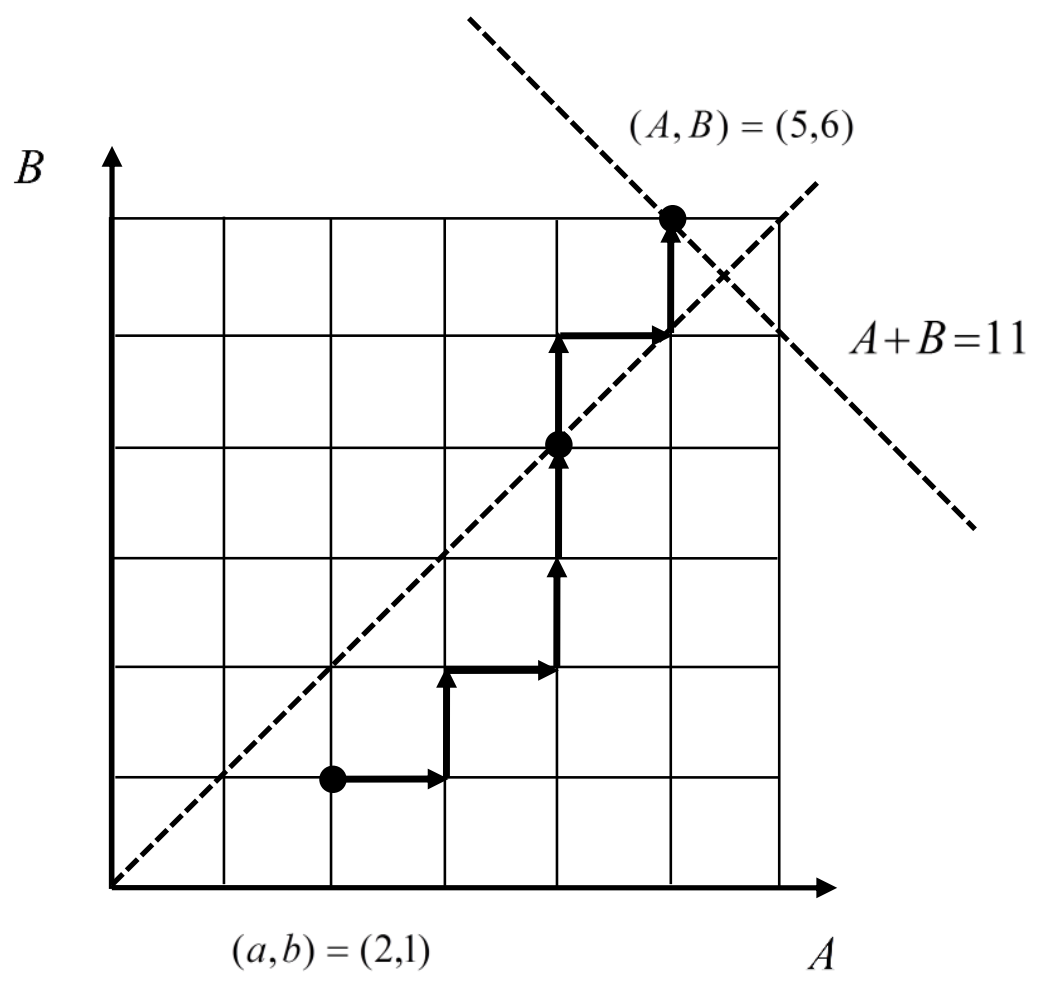

Figure 1. Illustrating a realization (directed lattice path) of the process. The path shown starts at $(a, b)=(2,1)$ and ends after $S=8$ steps at $(A, B)=(5,6)$ on the line $A+B=S+a+b=11$. The path first reaches the line $B=A$ at $(4,4)$. 


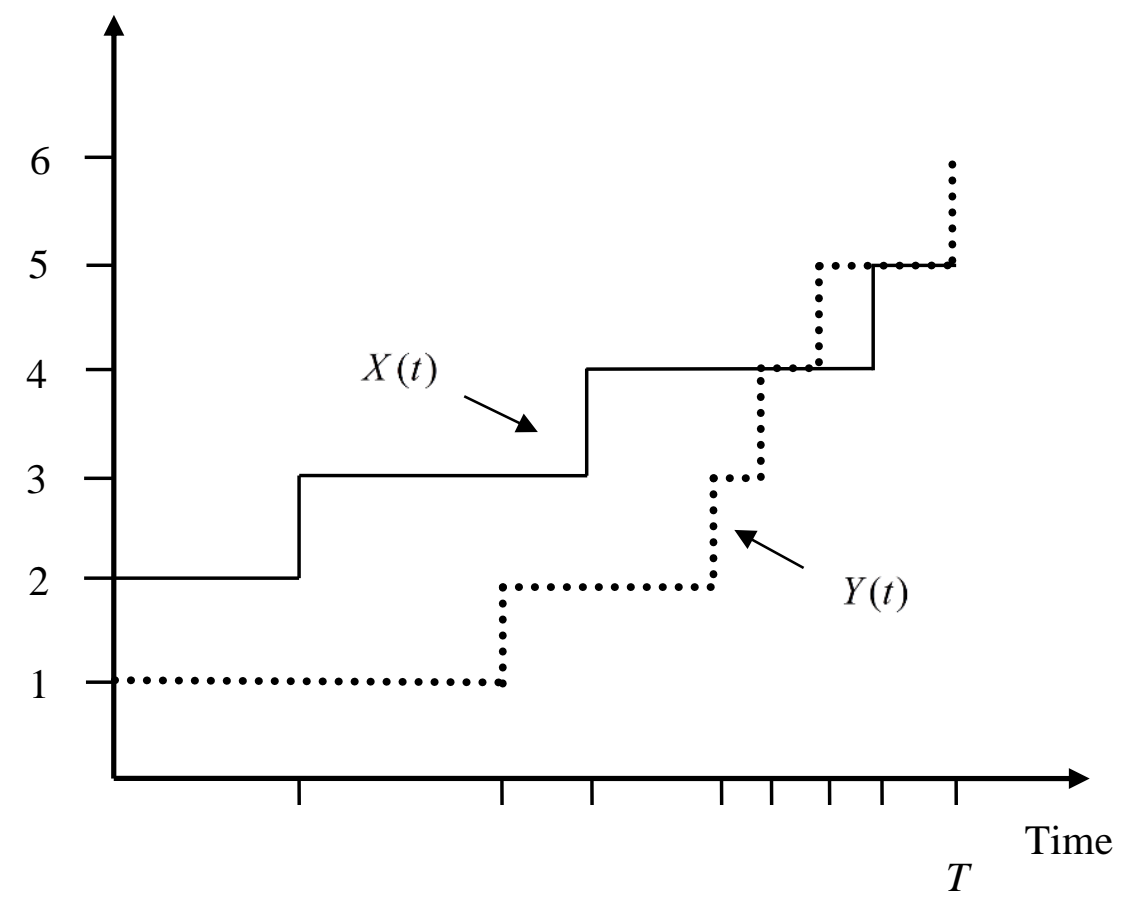

Figure 2. Illustrating the two independent birth processes $X(t)$ (solid line) and $Y(t)$ (dashed line) and an example of an ordered sequence of jumps $(X, Y, X, \ldots, Y)$ corresponding to the lattice path shown in figure 1 . The first passage time $T$ for the process $Y(t)$ to reach state $k=6$ is indicated. 


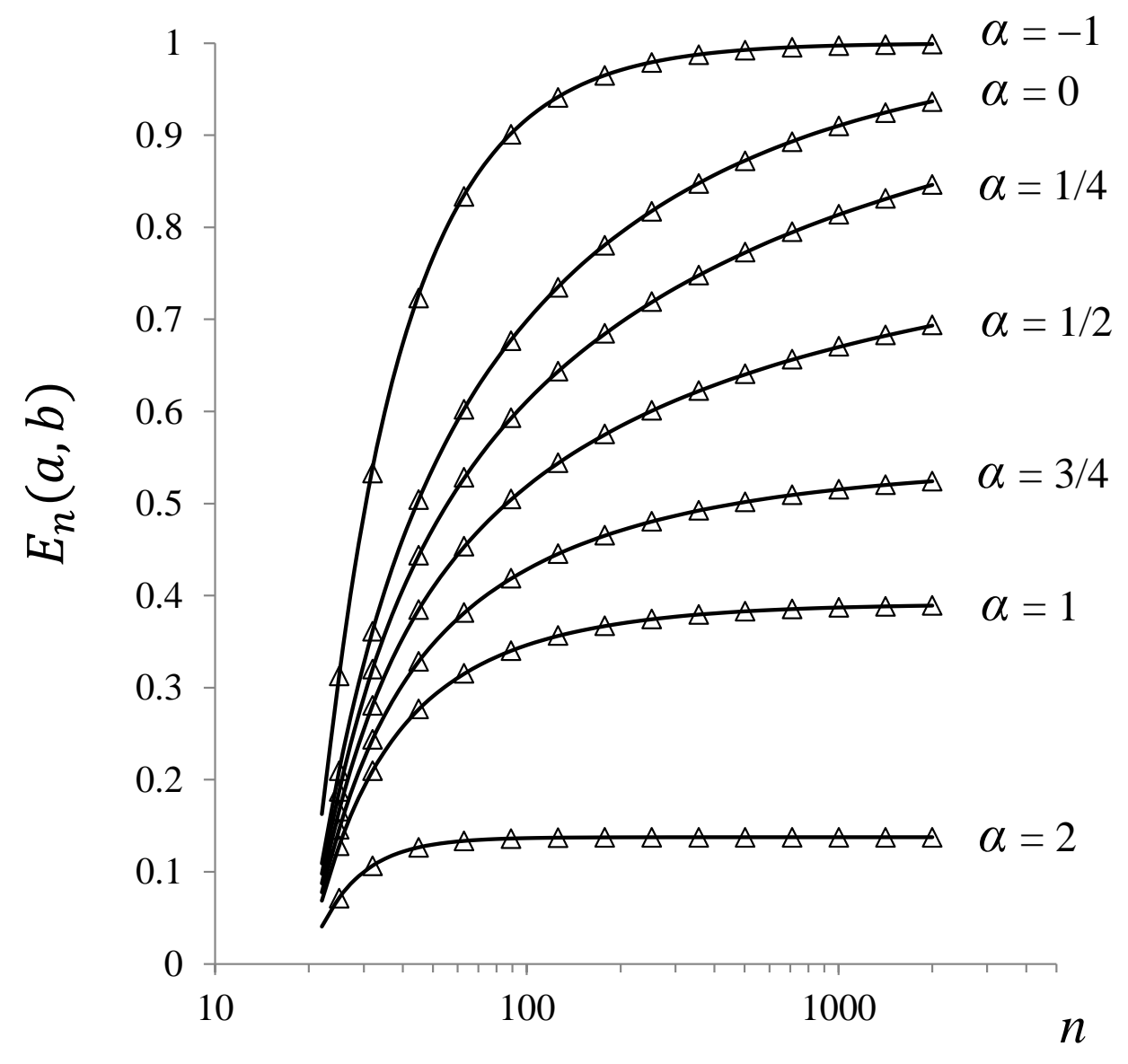

Figure 3. Comparison of the exact exit probability (triangles) with the results derived from simulations (solid lines) as a function of $n$ for various values of $\alpha$. The initial condition is $(a, b)=(20,15)$ in each case. 


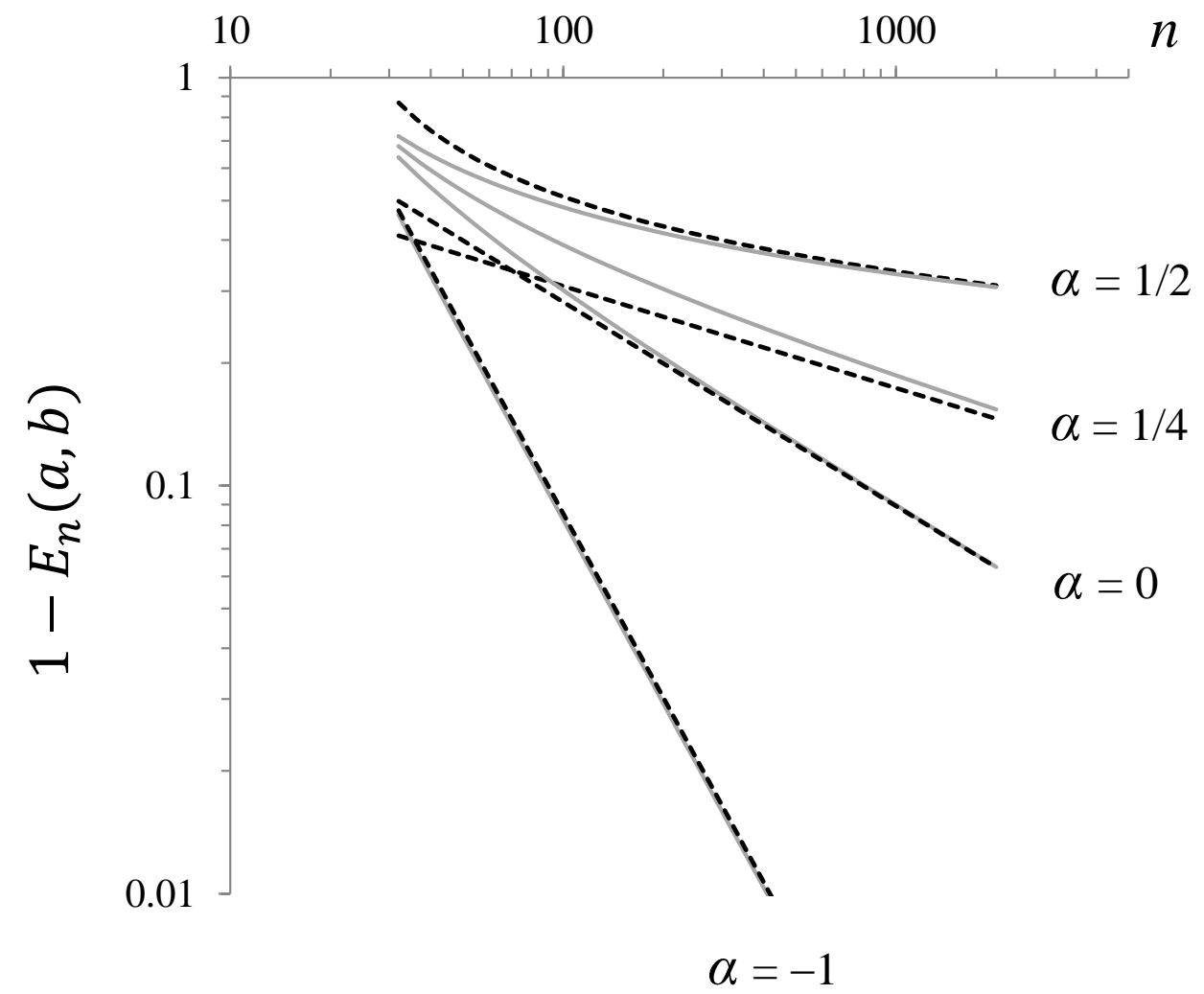

Figure 4. Comparison of the survival probability $\bar{E}_{n}(a, b) \equiv 1-E_{n}(a, b)$ as a function of $n$ for various values of $\alpha \leq \frac{1}{2}$ (solid lines) with the $n \rightarrow \infty$ asymptotes given by (17) (dotted lines). The initial condition is $(a, b)=(20,15)$ in each case. 


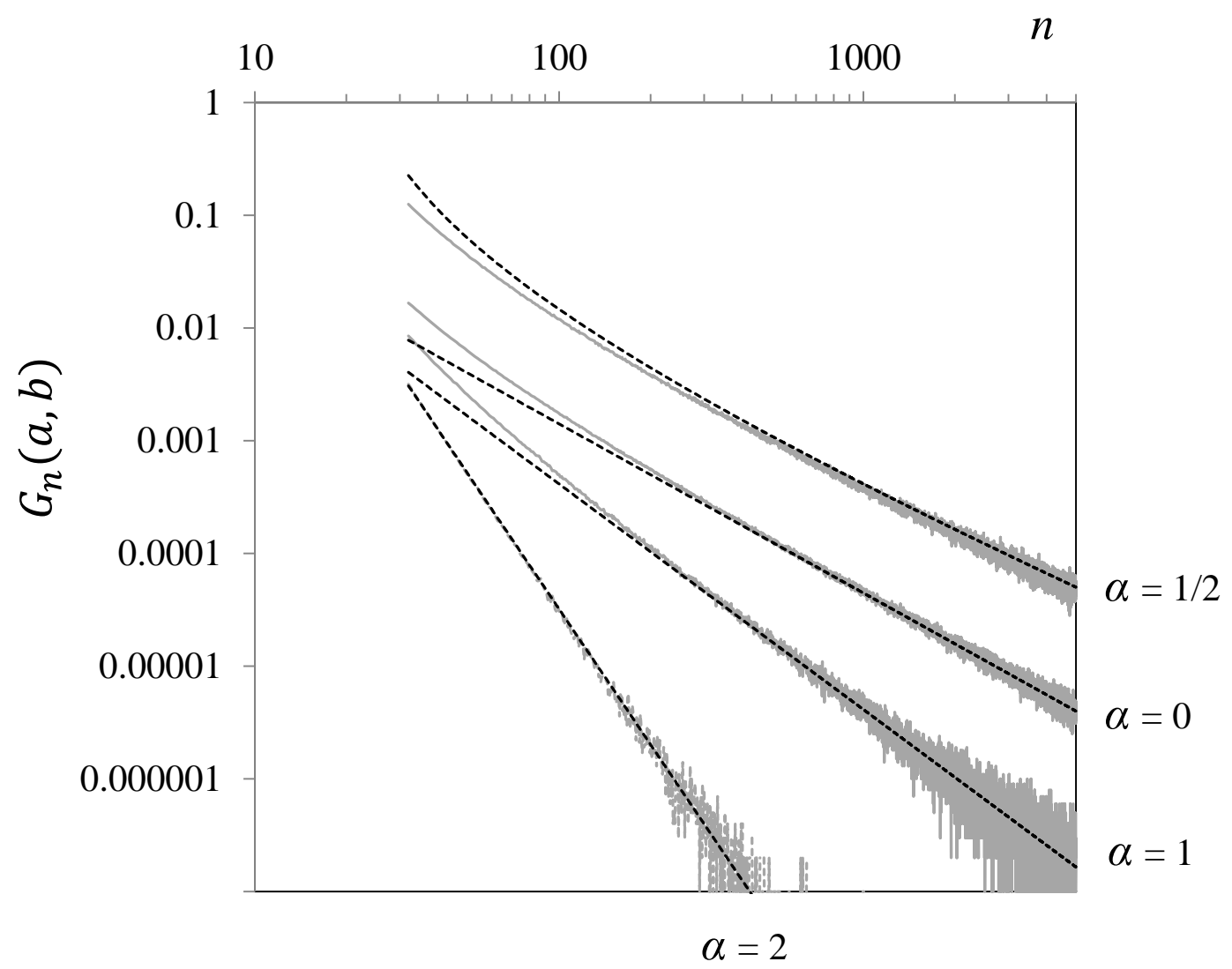

Figure 5. Comparison of the theoretical $n \rightarrow \infty$ asymptotes for the first passage probability given by (20) (dotted lines) with the results derived from simulations (solid lines) as a function of $n$ for various values of $\alpha$. The data for $\alpha=\frac{1}{2}$ have been displaced vertically by a factor of 10 for clarity. The initial condition is $(a, b)=(20,15)$ in each case 